\title{
Pleuroperitoneal Leak Complicating Continuous Ambulatory Peritoneal Dialysis: A Case Report
}

\author{
Sedat Ustundag, ${ }^{\mathrm{a}, \text { d }}$, Funda Ustun ${ }^{\mathrm{b}}$, Gulay Yilmaz ${ }^{\mathrm{a}}$, Erkan Narlic, Alp Avi Hararic
}

\begin{abstract}
Acute massive pleuroperitoneal leak-related hydrothorax is a rare, but life-threatening complication of continuous ambulatory peritoneal dialysis (CAPD). Hydrothorax develops right after the initiation of the CAPD or within a few days post-treatment. The late development of hydrothorax in patients treated with CAPD is quite rare. The interpretation of pleuroperitoneal leak-related hydrothorax as a volume overload and subsequent application of hypertonic peritoneal exchange worsen the clinical course and can lead to mortality. We here present the data from a patient who developed acute bilateral hydrothorax at the 16th month of CAPD treatment.
\end{abstract}

Keywords: Hydrothorax; Peritoneal dialysis; Tc99m-MAA scintigraphy

\section{Introduction}

End-stage renal disease (ESRD) is an increasing public health concern worldwide. As of 1980s, peritoneal dialysis got into the use in renal replacement therapy of ESRD patients as well as hemodialysis. The advantage of continuous ambulatory peritoneal dialysis (CAPD) compared with hemodialysis is the lower risk of blood borne diseases, the lack of need for systemic heparinization and better preservation of residual renal function. Since CAPD provides a flexible treatment independent of dialysis center, it is a desired treatment by patients [1].

Acute massive pleuroperitoneal leak-related hydrothorax is a rare, but life-threatening complication of peritoneal

\footnotetext{
Manuscript accepted for publication January 14, 2015

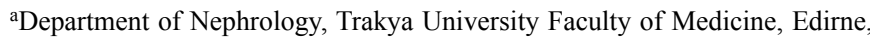
Turkey

bepartment of Nuclear Medicine, Trakya University Faculty of Medicine, Edirne, Turkey

${ }^{c}$ Department of Internal Medicine, Trakya University Faculty of Medicine, Edirne, Turkey

${ }^{\mathrm{d} C o r r e s p o n d i n g ~ A u t h o r: ~ S e d a t ~ U s t u n d a g, ~ T r a k y a ~ U n i v e r s i t e s i ~ T i p ~ F a k u l t e s i, ~}$ Nefroloji BD, 22030 Edirne, Turkey. Email: sedatustundag@yahoo.com
}

doi: http://dx.doi.org/10.14740/jmc2055w dialysis (PD) [2]. The prevalence of hydrothorax in ESRD patients treated with PD is reported to be approximately $2 \%$ [3]. In general, right-sided pleural effusion, transudative in nature develops right after the initiation of the PD or within a few days post-treatment. The late development of hydrothorax in patients treated with CAPD is quite rare. Dyspnea is the most important complication in patients with hydrothorax, and the extent of dyspnea depends on the severity of the pleuroperitoneal leak. The differential diagnosis of pleuroperitoneal leak-related hydrothorax should be made from the transudative effusion in congestive heart failure, hypoalbuminemia, and excessive volume overload [4]. The interpretation of pleuroperitoneal leak-related hydrothorax as a volume overload and subsequent application of hypertonic peritoneal exchange worsen the clinical course and can lead to mortality.

We here present the data from a patient who developed acute bilateral hydrothorax at the 16th month of CAPD treatment.

\section{Case Report}

A 43-year-old female, with end-stage renal failure secondary to chronic pyelonephritis, on CAPD for 19 months presented to the emergency room with acute dyspnea. Patient had approximately $1,000 \mathrm{~mL}$ urine; PD prescription consisted of four cycles of $2 \mathrm{~L}$ exchanges $(32 \mathrm{~mL} / \mathrm{kg}$ ) with $1.36 \%$ dextrose solution per day. One week before the onset of dyspnea, the patient moved house and actively participated in this move. Patient reported the presence of similar symptoms about 6 months ago when she was noncompliant with her sodium restricted diet. Thus, she tried to comply with sodium restricted diet and continued to have CAPD. She presented to the emergency room with nocturnal shortness of breath, and respiratory rate, blood pressure and pulse were measured 23/min, 138/83 $\mathrm{mm} \mathrm{Hg}$ and $74 /$ min and rhythmic, respectively. Patient weighed $69.2 \mathrm{~kg}$ (dry weight measured in last visit $63.5 \mathrm{~kg}$ ). Physical examination revealed bilateral lower extremity edema $(++)$, bilaterally decreased breath sounds more prominent in the right hemithorax and dullness to percussion in the right middle zone. Electrocardiogram was consistent with the previous recordings. Laboratory findings consisted of creatinine $6 \mathrm{mg} / \mathrm{dL}$, BUN 51 $\mathrm{mg} / \mathrm{dL}$, albumin $3.5 \mathrm{mg} / \mathrm{dL}$, sodium $140 \mathrm{mEq} / \mathrm{L}$, potassium $4.5 \mathrm{mEq} / \mathrm{L}, \mathrm{AST} 21 \mathrm{U} / \mathrm{L}$, and ALT $20 \mathrm{U} / \mathrm{L}$. In complete blood 


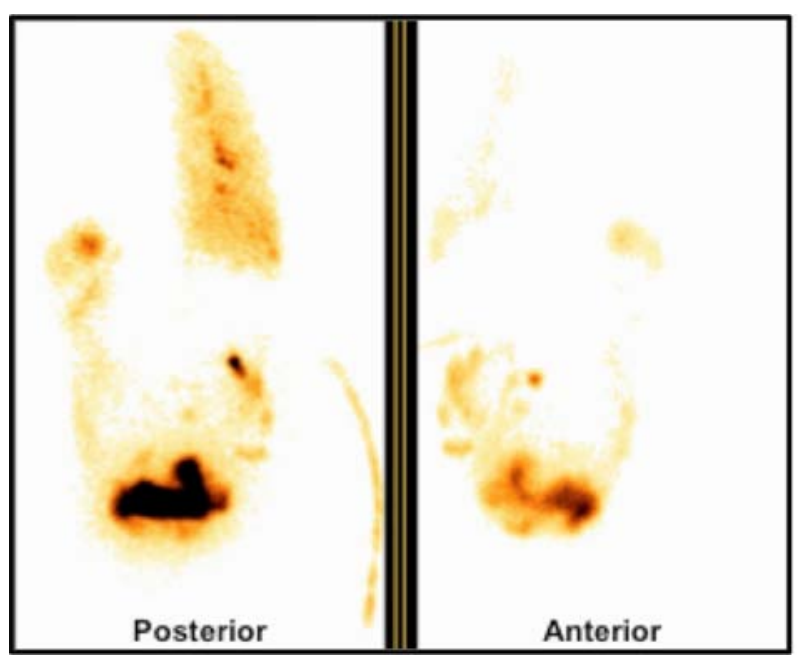

Figure 1. Scintigraphic images on admission.

count, WBC was $6,700 \mathrm{~mm}^{3}, \mathrm{Hb}$ was $11.1 \mathrm{~g} / \mathrm{dL}$, and PLT was 298,000 $\mathrm{mm}^{3} . \mathrm{pH}, \mathrm{HCO}_{3}$ and $\mathrm{pCO}_{2}$ were measured 7.4, 21 $\mathrm{mEq} / \mathrm{L}$ and $36 \mathrm{~mm} \mathrm{Hg}$ respectively in arterial blood gas analysis. The $\mathrm{P} / \mathrm{A}$ chest $\mathrm{X}$-ray revealed extensive right-sided pleural effusion and admitted to the hospital with the preliminary diagnosis of volume overload.

Patient complaining of weakness and fatigue, vomiting and cough productive of phlegm and reporting the relief of symptoms in the sitting position was administered $80 \mathrm{mg}$ of IV loop diuretic. The initial dialysate exchange was performed with 2,000 mL PD solution containing $2.7 \%$ glucose and patient's shortness of breath got worse. Diagnostic thoracentesis was performed and revealed pleural fluid glucose $439 \mathrm{mg} / \mathrm{dL}$, LDL $23 \mathrm{U} / \mathrm{L}$, and total protein $286 \mathrm{mg} / \mathrm{dL}$. Simultaneous to the thoracentesis, a sample of serum was obtained and glucose was measured $105 \mathrm{mg} / \mathrm{dL}$. In order to confirm the diagnosis of the pleuroperitoneal leak-related hydrothorax, scintigraphic imaging using a dose of $6 \mathrm{mCi}$ of technetium-99m macroaggregated albumin (Tc99m-MAA) was performed (Fig. 1). Dynamic and static SPECT images showed the presence of a pleuroperitoneal leak in left and right hemithorax, with leakage being evident in the right hemithorax. PD is discontinued. Since patient refused pleurodesis, permanent dialysis catheter was placed and hemodialysis was initiated. After 8 weeks of hemodialysis treatment, SPECT evaluation repeated for the patient who prefers to do PD (Fig. 2). Automated peritoneal dialysis (APD) was initiated after confirming the absence of pleuroperitoneal leak. The patient was followed up once a week, and then every 2 weeks and no hydrothorax observed.

\section{Discussion}

The fluid passage between the peritoneal and pleural cavity stems usually from the congenital or acute diaphragmatic defects $[4,5]$. It has been mooted that during the PD treatment, increased intraabdominal pressure can tear the previously intact collagen fibers and eventually leads to leakage [4]. In addition, it has been reported that the PD treatment in patients with

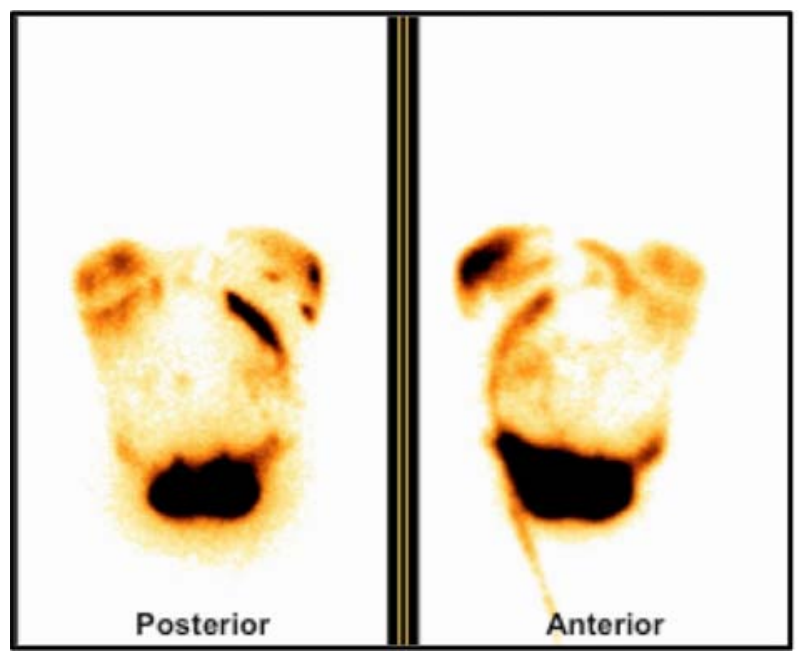

Figure 2. Scintigraphic images 8 weeks after cessation of PD.

disturbances in the lymphatic drainage can be the cause of the hydrothorax [6]. Krishnan et al [7] identified the frequent development of acute hydrothorax in patients on PD initiated at $40 \mathrm{~mL} / \mathrm{kg}$ dialysate volume compared to the patient's dialysate volume gradually increased. It is evident that acute hydrothorax can develop in few hours to few years in patients having CAPD $[5,8,9]$. Pleuro-peritoneal leak secondary to congenital defect usually develops shortly after the initiation of the PD treatment $[5,9]$. However, like in our case, there are patients presenting with the late development of hydrothorax even in the eighth year of PD treatment [2]. Hydrothorax usually develops in the right side since left diaphragmatic defects are covered by heart and pericardial surface [5, 8-11].

In cases developing hydrothorax, depending on the severity of the leak, majority of the cases present with sudden-onset dyspnea as well as asymptomatic cases $[4,5,8,11]$. Dyspnea is more prominent at night and it resolves in the sitting position. Pleura-peritoneal leak-related hydrothorax cases can present with ultrafiltration failure, discomfort in the chest and cough. Physical examination consists of unilateral (leak side) decrease in breath sounds, increase in thoracic vibrations and dullness to percussion. P/A chest X-ray demonstrates leak side pleural effusion. Congestive heart failure, hypoalbuminemia, tuberculous pleurisy and ultrafiltration failure should be considered in the differential diagnosis of pleuroperitoneal leakrelated hydrothorax [12]. Misdiagnosing hydrothorax and treating this condition as an ultrafiltration failure and increasing the dialysate osmolarity can cause worsening of clinical course [13].

Biochemical examination of the pleural fluid in cases developing hydrothorax secondary to pleuro-peritoneal leakage is guiding for the clinician. Pleural fluid is in transudative in nature and protein and LDH concentration is lower than the serum; in contrast pleural fluid glucose concentration is higher than the serum glucose concentration and therefore called "sweet" hydrothorax $[2,5,10]$. The diagnostic cut-off value of the pleural glucose concentration is obscure. Even though pleural fluid glucose concentration is recommended to be $>300$ - $400 \mathrm{mg} / \mathrm{dL}$ in terms of diagnosis [14], there are pleuro-perito- 
neal leakage cases reported with the pleural fluid glucose concentration of $123 \mathrm{mg} / \mathrm{dL}$ [12]. In order to diagnose the leakage, Chow et al [13] suggested to use pleural fluid-to-serum glucose gradient and $>50 \mathrm{mg} / \mathrm{dL}$ gradient being the cut-off value rather than using pleural fluid glucose concentration. However, even in less gradient change, it has been reported that pleural fluid collection can be secondary to pleura-peritoneal leakage $[5,12]$. The glucose content of pleural fluid depends on the diameter of the defect causing the leakage, absorption rate of glucose from the pleural surface, glucose concentration of the dialysate and the duration between the dialysate exchange and pleural fluid sample collection [5]. Variations of values in all these studies probably result from the above mentioned factors. Therefore, a precise cut-off value for the pleural fluid glucose concentration and the pleural fluid-to-serum glucose gradient could not be determined. Demonstration of the presence of D-lactate in the pleural fluid, a molecule not synthesized in human body and used to buffer peritoneal dialysis fluids, is beneficial in terms of diagnosis. Lack of analysis of this lactate isomer in majority of the centers limits its usage [6].

Radionuclide scanning is a trusted method in the diagnosis of the pleuro-peritoneal leakage $[4,5,10,12]$. In order to visualize the leakage $3-10 \mathrm{mCi}$ dose of Tc99m-MAA or sulfur colloid is added to the dialysate and periodic images from 30 $\min$ to $4 \mathrm{~h}$ are obtained. Activity in the thorax is considered the proof of leakage.

In patients identified with pleuro-peritoneal leakage, as the initial step of the treatment PD should be paused and symptomatic relief should be provided with the help of thoracentesis. Secondly, repair of the defect causing the leakage could be performed. Suspending PD treatment for 2 - 8 weeks and temporary hemodialysis during this period is the frequent treatment of choice $[3,14]$. Removal of the pressure gradient between peritoneum and pleura can lead to healing of the diaphragmatic defect causing the leakage. Because temporary interruption of CAPD risk-free, it is generally recommended as the first-line treatment option. This approach results in recovery in $50 \%$ of the cases, but there is a high rate of recurrence [3]. Another option is the surgical closure of the defect causing the leakage with conventional pleurodesis, open thoracotomy or videoassisted thoracoscopy (VATS). In conventional pleurodesis, sclerosing agents like talc, autologous blood, glue, bleomycin, doxycycline or tetracycline can be infused after the insertion of a thorax tube into the hemithorax with pleuroperitoneal leakage. Fibrosis resulting from the inflammation caused by a sclerosing agent can permanently patch the defect causing the leakage [3, 4]. It is adequate to wait for 10 days prior to resuming PD treatment in cases having pleurodesis. It should be bear in mind that pleurodesis is a painful treatment and there is $50 \%$ recurrence rate [3]. In open thoracotomy, defect in the diaphragm is accessed through a small intercostal incision. Diaphragm is directly visualized and other possible defects can be identified with the concurrent infusion of fluid into the peritoneum. Defect can be repaired with sutures and Teflon patch may be used to reinforce the repair. After the repair, infusion of fluid into the peritoneum helps identification of any remnant leakage. In cases repaired by open thoracotomy, no recurrence has been reported $[3,15]$. VATS can be utilized in the treatment since it enables minimally invasive visualization of the diaphragm, repair of the potential defect or infusion of the sclerosing agent directly into the defective region. In patients treated with thoracoscopic pleurodesis, PD treatment can be restarted in 3 - 4 weeks post pleurodesis with a very low rate of recurrence $[3,16]$. On the other hand, it has been shown that thoracoscopic diaphragm plication allows starting peritoneal dialysis on the week of operation [17].

In conclusion, development of acute hydrothorax in end-stage renal failure patients on CAPD is a rare, but lifethreatening complication. Major complaint of the patients is the acute dyspnea, exacerbating at night and resolving in the sitting position. Diagnosis is made by the demonstration of the increased glucose content of the pleural fluid compared to serum in biochemical examination and identification of the leakage using the imaging techniques. Discontinuation of PD treatment $2-8$ weeks for the self-healing of the diaphragmatic defect or repairment of the defect with conventional pleurodesis, open thoracotomy, VATS allows patients to resume PD treatment after the complicating leakage.

\section{Conflict of Interest}

There is no conflict of interest.

\section{References}

1. Ghaffari A, Kalantar-Zadeh K, Lee J, Maddux F, Moran J, Nissenson A. PD First: peritoneal dialysis as the default transition to dialysis therapy. Semin Dial. 2013;26(6):706713.

2. Nomoto Y, Suga T, Nakajima K, Sakai H, Osawa G, Ota $\mathrm{K}$, Kawaguchi Y, et al. Acute hydrothorax in continuous ambulatory peritoneal dialysis--a collaborative study of 161 centers. Am J Nephrol. 1989;9(5):363-367.

3. Chow KM, Szeto CC, Li PK. Management options for hydrothorax complicating peritoneal dialysis. Semin Dial. 2003;16(5):389-394.

4. Szeto CC, Chow KM. Pathogenesis and management of hydrothorax complicating peritoneal dialysis. Curr Opin Pulm Med. 2004;10(4):315-319.

5. Tapawan K, Chen E, Selk N, Hong E, Virmani S, Balk R. A large pleural effusion in a patient receiving peritoneal dialysis. Semin Dial. 2011;24(5):560-563.

6. Garcia Ramon R, Carrasco AM. Hydrothorax in peritoneal dialysis. Perit Dial Int. 1998;18(1):5-10.

7. Krishnan RG, Ognjanovic MV, Crosier J, Coulthard MG. Acute hydrothorax complicating peritoneal dialysis. Perit Dial Int. 2007;27(3):296-299.

8. Conde Rivera O, Camba Caride M, Iglesias Lamas E, Perez Melon C. Hydrothorax in peritoneal dialysis: a rare complication. Nefrologia. 2011;31(1):110-112.

9. Wang HB, Kao CC, Hsu KF, Yang MH, Yu HC, Chen JC. Diaphragmatic bleb complicated hydrothorax in peritoneal dialysis. Intern Med. 2009;48(15):1333-1334.

10. Gidaris D, Printza N, Batzios S, Belechri AM, Papachristou F. A "sweet" hydrothorax in a child on peritoneal di- 
alysis. Hippokratia. 2011;15(4):358-360.

11. Smolin B, Henig I, Levy Y. "Sweet" hydrothorax complicating chronic peritoneal dialysis. Eur J Intern Med. 2006;17(8):583-584.

12. Huang JJ, Wu JS, Chi WC, Lan RR, Yang LF, Chiu NT. Hydrothorax in continuous ambulatory peritoneal dialysis: therapeutic implications of Tc-99m MAA peritoneal scintigraphy. Nephrol Dial Transplant. 1999;14(4):992997.

13. Chow KM, Szeto CC, Wong TY, Li PK. Hydrothorax complicating peritoneal dialysis: diagnostic value of glucose concentration in pleural fluid aspirate. Perit Dial Int. 2002;22(4):525-528.
14. Leblanc M, Ouimet D, Pichette V. Dialysate leaks in peritoneal dialysis. Semin Dial. 2001;14(1):50-54.

15. Allen SM, Matthews HR. Surgical treatment of massive hydrothorax complicating continuous ambulatory peritoneal dialysis. Clin Nephrol. 1991;36(6):299-301.

16. Mak SK, Chan MW, Tai YP, Wong PN, Lee KF, Fung LH, Wong AK. Thoracoscopic pleurodesis for massive hydrothorax complicating CAPD. Perit Dial Int. 1996;16(4):421-423.

17. Saito M, Nakagawa T, Tokunaga Y, Kondo T. Thoracoscopic surgical treatment for pleuroperitoneal communication. Interact Cardiovasc Thorac Surg. 2012;15(4):788789 . 